\title{
Detectability of possible space weather effects on Mars upper atmosphere and meteor impacts in Jupiter and Saturn with small telescopes
}

\author{
Ricardo Hueso ${ }^{1, *}$, Teresa del Río-Gaztelurrutia ${ }^{1}$, Agustín Sánchez-Lavega ${ }^{1}$, Marc Delcroix ${ }^{2}$, \\ Jon Juaristi ${ }^{1}$, and Luis Carlos Fernández-González ${ }^{1,3}$ \\ 1 Dpto. Física Aplicada I, Escuela de Ingeniería de Bilbao, UPV/EHU, Bilbao, Spain \\ 2 Commission des Observations planétaires, Sociéte Astronomique de France, Paris, France \\ 3 Satlantis, Parque Científico UPV/EHU, Leioa, Spain
}

Received 3 June 2018 / Accepted 18 November 2018

\begin{abstract}
Amateur astronomers operating small telescopes accumulate a larger amount of observational time of Solar System planets than the ensemble of professional telescopes. Over the last 15 years, advancements in low-cost fast acquisition cameras and image software processing tools have pushed forward the scientific analysis of these observations thanks to a sustained increase in quality and spatial resolution. The high temporal coverage attained by amateur astronomers collaborating with scientists allows detecting rare events in the atmospheres of different planets. In particular, high-atmosphere phenomena in Mars, possibly caused by solar activity, and meteor impacts in Jupiter have been observed serendipitously in the last decade. Here we review the contribution of amateur astronomers to these two fields. Coronal Mass Ejections arriving to Mars under favorable observing conditions can be predicted after observations of the Sun, and their possible effect on Mars upper atmosphere can be monitored with small telescopes. Impacts in Jupiter of two different classes can be discovered by amateur astronomers: intermediate size impacts big enough to leave a debris field on its atmosphere (150-m size objects before the impact), and fireballs caused by objects of 5-20 m producing short-lived flashes in video observations of the planet. In this regard, we present a software tool designed to analyze video observations of Jupiter and Saturn that should help to find new impact flashes on the giant planets.
\end{abstract}

Keywords: Mars / Sun: coronal mass ejections (CMEs) / Jupiter / meteors, meteoroids

\section{Introduction}

Amateur astronomers operating small-size telescopes contribute to the regular observation of the Solar System with high-quality data and with far more observing time than the combination of all professional telescopes. The involvement of many amateur observers in scientific studies of the Solar System is experiencing a large increase in terms of number of publications and range of topics (Mousis et al., 2014). Advancements in low-cost amateur instrumentation have produced notable results in terms of data quality. In particular, amateur astronomers regularly obtain high-resolution images of the planets using a variant of the lucky-imaging technique (Law et al., 2006; Mendikoa et al., 2014). Fast video cameras are used to observe the planets obtaining thousands of short

\footnotetext{
${ }^{*}$ Corresponding author: ricardo.hueso@ehu.eus
}

exposure frames that freeze the effects of the atmospheric seeing. The best frames are selected by software tools popular in the amateur community such as AutoStakkert (Kraaikamp, 2016) and stacked into high signal to noise ratio images. The stacks are processed with either high-pass filters or wavelet sharpening software resulting in enhanced images (Mousis et al., 2014). Consequently, public repositories of amateur images of the planets are now widely used by professional astronomers to complement or guide their observations with professional telescopes (Hueso et al., 2010a, 2018a). One of the key contributions of amateur astronomers is their capacity to obtain large amounts of observations sampling the timedomain with a high temporal cadence from the collaboration of many individual observers. Therefore, amateur astronomers can discover unexpected or unpredictable events in Solar System science serendipitously even though many planets have dedicated space missions orbiting them. For instance, the onset 
of atmospheric disturbances in the giant planets is generally discovered by amateur astronomers (Mousis et al., 2014 present several examples). Here we cover two very different topics related with possible space weather in Mars and impacts in Jupiter with a common link in the fundamental contribution to both topics from amateur astronomers.

In March and April 2012 two bright, extremely highaltitude plumes were observed by amateur astronomers at the Martian terminator (the day-night boundary) over a period of 10 days in each event. The altitude of these features was 200-250 km above the surface, and, thus, well into the ionosphere and the exosphere (Sánchez-Lavega et al., 2015). Their occurrence has been proposed to be caused by space weather effects linked to the arrival of Coronal Mass Ejections (CMEs) to Mars (Andrews et al., 2016). Although this possibility remains not fully demonstrated, other options, like upwelling of dust from the surface, condensing clouds or exogenous interplanetary dust seem even less likely (Andersson et al., 2015; Sánchez-Lavega et al., 2015) and more observations of these type of events are required to understand their nature.

In July 2009 Jupiter was hit by an object with an estimated size of 500-m (Sánchez-Lavega et al., 2010) that left a dark debris field on the atmosphere. This impact was first observed by an amateur astronomer, Anthony Wesley (Australia), and quickly followed-up by Hubble Space Telescope (HST) observations (Hammel et al., 2010) and a range of large telescope facilities (de Pater et al., 2010). The impact was fully unexpected and its occurrence contradicted predicted impact rates on Jupiter considered correct at the time of the Shoemaker-Levy 9 (SL9) impact in 1996 (Harrington et al., 2004). A year later, the same amateur astronomer observed a short (1 s) bright flash on Jupiter produced by a small size impact (Hueso et al., 2010b). Four more flashes have been observed in Jupiter since then. These flashes are considered to be caused by impacts of objects with diameters from 5 to $20 \mathrm{~m}$ (Hueso et al., 2013, 2018 b). The combined analysis of the ensemble of these observations results in an increased estimation of the impact rate with respect to previous works (Sánchez-Lavega et al., 2010; Hueso et al., 2013, 2018b).

Unexpected events like these (high plumes on Mars, dark debris fields on Jupiter and flashes on Jupiter video observations) cannot be predicted and their discovery requires large amounts of observing time that are generally only available to the ensemble of amateur astronomers observing the planets. Flashes on Saturn video observations caused by impacts slightly larger than those in Jupiter are also potentially luminous enough to be discovered with the same techniques. Here we examine the detectability of such events with the help of amateur astronomers that regularly observe the planets using small telescopes. We also discuss ways to maximize the outcome of such observations.

\section{Mars high altitude plumes}

Mars surface dust and condensate clouds of water and $\mathrm{CO}_{2}$ ice can reach altitudes up to $\sim 50-100 \mathrm{~km}$ above the surface. Their vertical structure, aerographic location and seasonal occurrence, have been analyzed using data from instruments onboard spacecrafts orbiting Mars (Clancy et al., 2017; Wolff et al., 2017). Clouds and dust have also been imaged by orbiters as detached layers on the limb and terminator (Jaquin et al., 1986; Santer et al., 1986; Clancy et al., 2007; Sánchez-Lavega et al., 2018), and also as "protrusions" on the limb and terminator with the HST (Pellier, 2012; Sánchez-Lavega et al., 2015) and from ground-based telescopes (Minami, 2003).

Figure 1 shows several instances of structures located high in the Martian atmosphere. An exceptional event occurred on Mars 2012 when 18 different amateur observers captured an elevated, large projection at the terminator with a "plume-like" aspect (Fig. If and g) (Sánchez-Lavega et al., 2015). The images showing the high plume were obtained at optical wavelengths with red, green and blue filters using telescopes with apertures in the range $20-40 \mathrm{~cm}$. The telescopes were equipped with fast cameras using the lucky-imaging technique in some cases allowing several observations over timescales of tens of minutes. The plume was observed as it appeared in the morning terminator as the planet was rotating, thus allowing a very precise estimation of its altitude which was $200-250 \mathrm{~km}$. This extremely high altitude is well into the ionosphere and reaches the exosphere of Mars making unreasonable a cloud formation as the origin of the high plume. The plume was not detectable when passing through the central meridian, and it could not be seen in the afternoon limb, indicating a daily evolution (Sánchez-Lavega et al., 2015). The event was observed during several days on Mars morning terminator above Terra Cimmeria, a region where the weak Martian magnetic field shows an anomaly with (Mitchell et al., 2007). The high-atmosphere structure visible in the images extended 500-1000 km (EastWest and North-South) and exhibited a day-to-day variability, lasting for 10 days in Mars 2012 and then reappearing for other 10 days in April 2012.

The origin of these remarkable phenomena remains uncertain. Photometric models indicate that small-size particles $(0.1 \mu \mathrm{m}$ in size) could produce the observed brightness and spectral reflectance if composed of ice (preferred to dust). However, neither ice, nor dust are expected to form or be lifted in the atmosphere to this high altitude (Sánchez-Lavega et al., 2015). Alternatively, if the plume resulted from emission phenomena in an aurora-like process, at the location of an anomaly of the Martian crustal magnetic field (Mitchell et al., 2007), it would imply an aurora in Mars orders of magnitude brighter than auroras in Earth. Additionally, if the plume resulted from dust deposited by the destruction of a bolide in the upper atmosphere, or by a higher concentration of interplanetary dust, there is no reason why there were two events extended in time and why they were observed in the morning but not in the evening.

Andrews et al. (2016) pointed to a possible connection of these events with the impact of Solar CMEs in Mars, an interpretation that had been already proposed for a similar event detected in 2003 by amateur astronomers (Minami, 2003). Andrews et al. (2016) reported plasma observations by two instruments onboard Mars Express (MEx): the Analyzer for Space Plasmas and Energetic Atoms, ASPERA-3 (Barabash et al., 2006) and the Mars Advanced Radar for Subsurface and Ionospheric Sounding, MARSIS (Picardi et al., 2004; Gurnett et al., 2005). These observations were simultaneous to the events in 2012, and both instruments showed a disturbed 


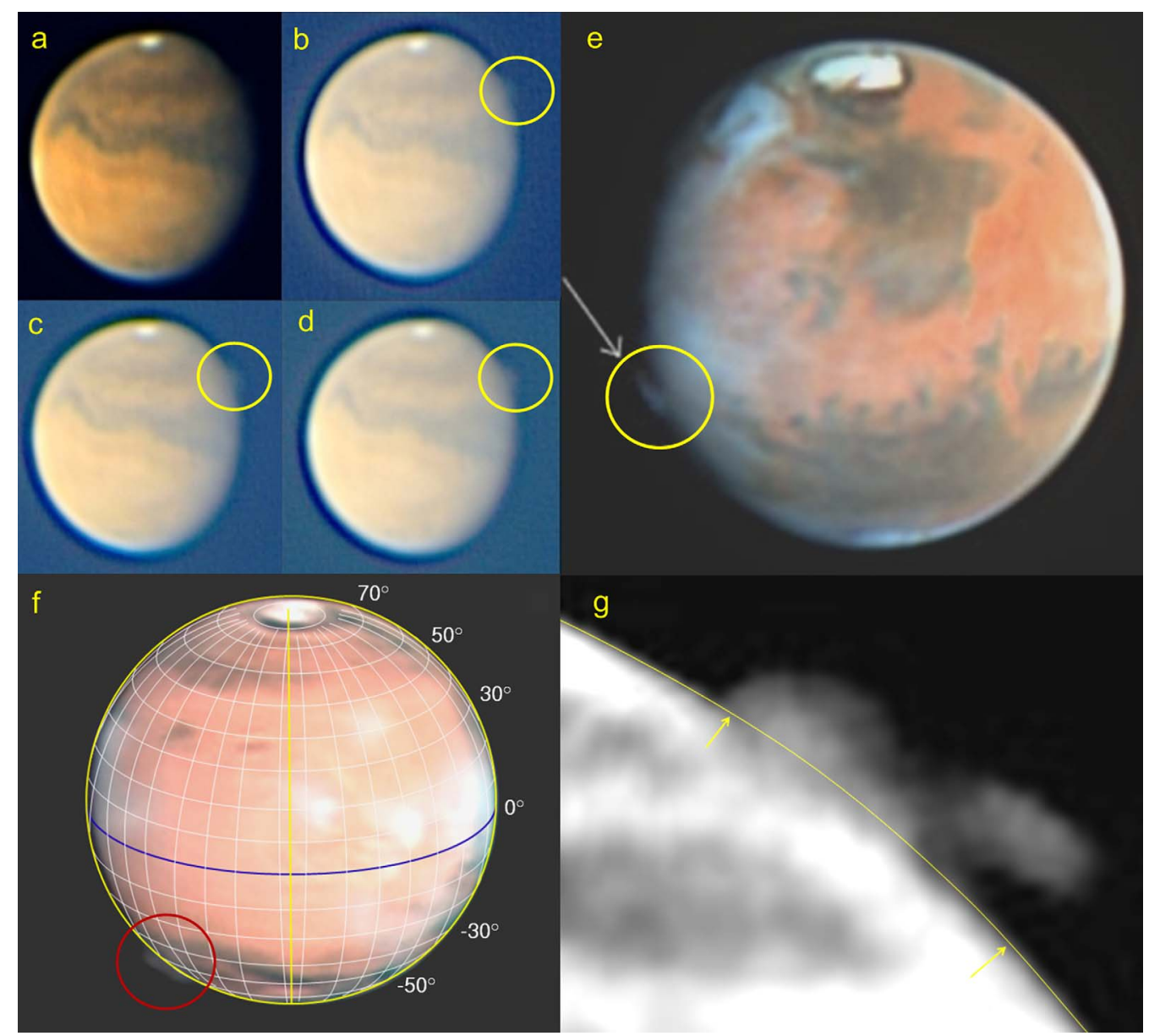

Fig. 1. Plume-like events marked on Mars terminator: (a)-(d) CCD images from Isao Miyazaki on 8 November 2003 at (a) 11:23 UT, (b) 11:23 UT (enhanced), (c) 11:41 UT (enhanced), (d) 12:00 UT (enhanced) (from Mimami, 2003), (e) HST on 17 May 1997 (from Sánchez-Lavega et al., 2015), (f)-(g) CCD lucky-imaging from D. Parker on 21 March 2012 with an enlarged version of the plume event in (g) (from Sánchez-Lavega et al., 2015).

ionosphere, most likely caused by the arrival at Mars of a series of CMEs, the largest of which reached Mars on March 9, 3 days before the first report of the presence of the plume. Due to the geometry of the MEx orbit, these observations took place when the Region of Interest (ROI) was in the afternoon terminator, where observations from Earth did not detect the presence of any visible feature.

However, the connection of CMEs with the high-altitude phenomena observed in 2012 remains a working hypothesis. Although the mechanism that could create the observed plume due to the impact of a CME is unknown, it is of great interest to check the possible correlation between the two phenomena in possible future events. If a $\mathrm{CME}$ is discovered propagating to Mars, amateur astronomers can rapidly react to an alert obtaining observations of the planet. Alternatively, if amateur astronomers discover another high-plume event, then observations of the plasma environment and ionosphere of Mars gathered by MEx, Mars Atmosphere and Volatile EvolutioN (MAVEN) or other spacecraft could prove the connection. CME alerts are routinely launched by space weather agencies. At Martian oppositions, the likelihood that a CME that impacts the Earth also hits Mars is enhanced and current online services able to study the propagation of CMEs in the inner solar system can be also used. The fact that the visible plumes persisted for days is consistent with the time scales of other phenomenologies associated with the impact of CMEs on Mars (Duru et al., 2017; Thampi et al., 2018). The uncertainties in models of CME propagation through the inner Solar System suggest that an observational program aimed to produce alerts should suggest observations executed over several days requiring the cooperation of amateur astronomers.

Figure 2 explores the observational conditions of Mars comparing with the Solar cycle. Mars observing periods occur every two Earth years and the Mars opposition in April 2014 occurred with the solar maximum activity with no reports of high-plumes in the Martian atmosphere. Recent CMEs arriving to Mars on February-March 2015 have been characterized from observations obtained by MEx, MAVEN) and Mars Odissey (MO) (Duru et al., 2017; Thampi et al., 2018). However, these events have not been observed from Earth due to the orbital positions of Earth and Mars, or from orbital images sampling Mars limbs. Mars opposition in 2016 occurred near solar minimum and again no similar activity to the events found in 2012 are present in amateur images 


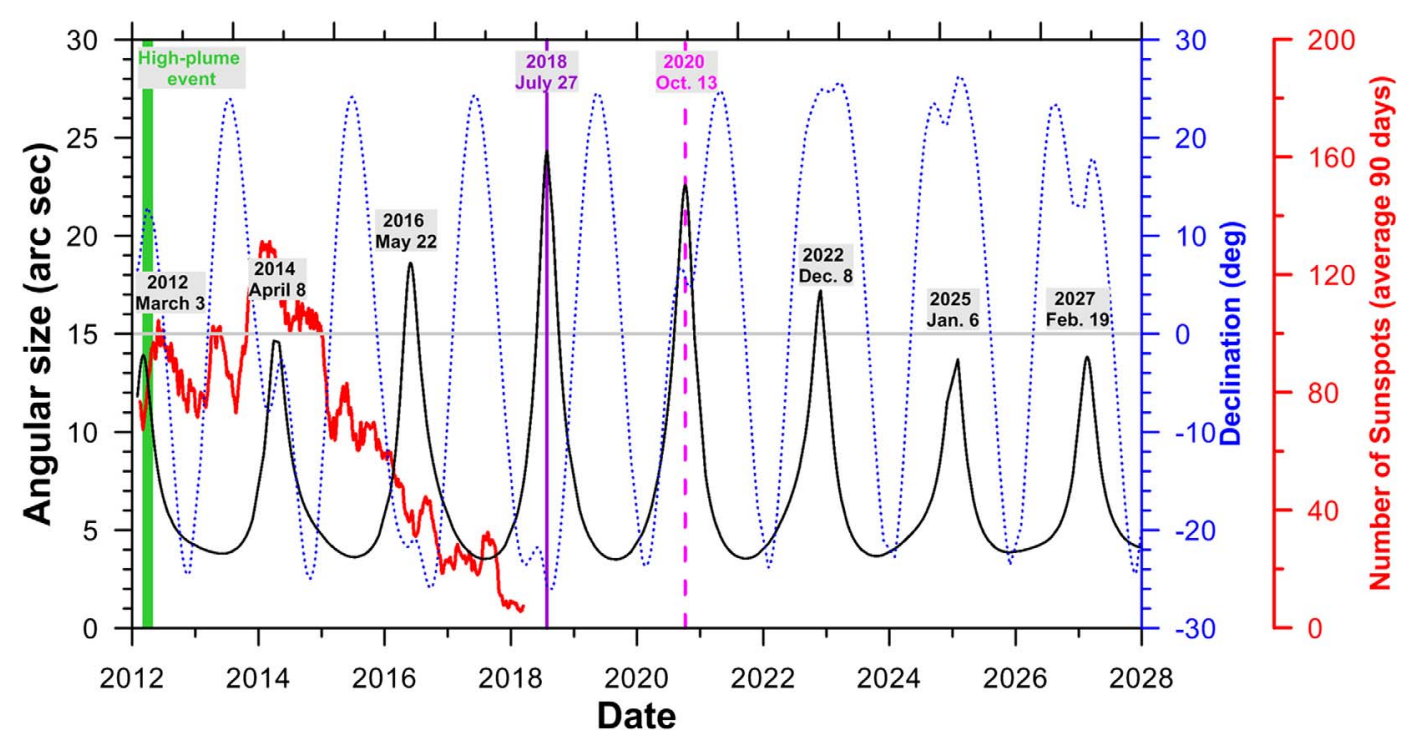

Fig. 2. Mars observational periods since the 2012 event up to 2028. Mars apparent angular diameter (left axis, black line) and planet declination (right axis, blue dashed line) mark the observational period of the planet. Minimums in the black curve show solar conjunction periods where no observations from Earth can be obtained. Dates of Martian oppositions when the planet shows a bigger size are highlighted. The last and next Mars oppositions are ideal for South and North hemisphere observers respectively. The connection with the solar activity is shown through the average number of sunspots (red line, red right axis). The high-plume event discussed in the text occurred in March-April 2012 (green vertical area on the left). The last and next Mars oppositions are shown in magenta and pink lines.

gathered during that period. Although the last Martian opposition (July 2018) was well suited for ground-based observations, the low level of solar activity resulted in a low probability to make such a connection. To our knowledge, no CME has arrived to Mars over the months close to the last opposition.

The Space Situation Awareness (SSA) program of the European Space Agency (ESA) provides unified access to some tools that may help observers determine if a CME impact is expected on Mars. Among them, alerts of CMEs and halo CMEs are issued by the Solar Influences Data Analysis Center of the Royal Observatory of Belgium (ROB/SIDC). The Heliospheric Weather Expert Service Centre of the UK Meteorological Office (HELSWE) provides access to model predictions of CME propagation in the heliosphere up to Mars using ENLIL (Odstrcil, 2003), a large scale, physics-based prediction model of the heliosphere. Additionally, if a plume is observed, the SSA program provides tools that allow checking if transients in the solar wind have reached Mars previously to the event. In particular, the Heliospheric Weather Expert Service Centre Propagation Tool (PROPTOOL; Rouillard et al., 2017) developed by the Centre de Données de la Physique des Plasmas (CDPP), allows easy access to data from different space probes and provides graphic tools (see Fig. 3) that permit a characterization of solar wind transients and a calculation of estimated times of arrival of CME to planets and probes.

Other spaces agencies provide also space weather alerts and prediction tools. The NOAA Space Weather Prediction Center (SPWC) gives access to a complete set of alerts and forecasts, and NASA's Integrated Space Weather Analysis System (iSWA), provides a very flexible and easy to use web-based system that gives access to space weather information. In particular, iSWA provides access to the "Ensemble CME
WSA-ENLIL + Cone Predict Summary" (Pizzo et al., 2011) that gives a summary of the results of a collection of WSAENLIL + Cone simulations. These simulations can be used to estimate the probability that a given CME hits a particular probe or planet. The tool also estimates the time of arrival in case hits are predicted. An example of a WSA-ENLIL + Cone simulation, run through the Community Coordinated Modelling Center (CCMC), is shown in Figure 4. Table 1 provides a list of the web services and tools discussed in the text.

\section{Jupiter impacts}

\subsection{Large impacts producing observable atmospheric debris and their detectability}

The SL9 series of impacts in Jupiter in July 1994 (Hammel et al., 1995; Harrington et al., 2004) demonstrated that planetary impacts can be observed safely on other planets from telescopic observations. The SL9 impacts have been studied in hundreds of scientific papers (see Harrington et al., 2004 for a review) and attracted observations from most telescopes on Earth including amateur observations with small telescopes. At the time, most amateurs reported drawings of the impacts (Pujic, 1994) but some of them were already using CCD cameras producing scientific images at moments in time not observed from professional observatories. Studies combining professional and amateur observations tracked the impact debris fields for months and the dispersal of the comet material in Jupiter's high atmosphere (Sánchez-Lavega et al., 1995).

The amateur involvement in the scientific outcome of the SL9 event was small, but 15 years later, the unexpected discovery by A. Wesley, an Australian amateur observer, of a second 


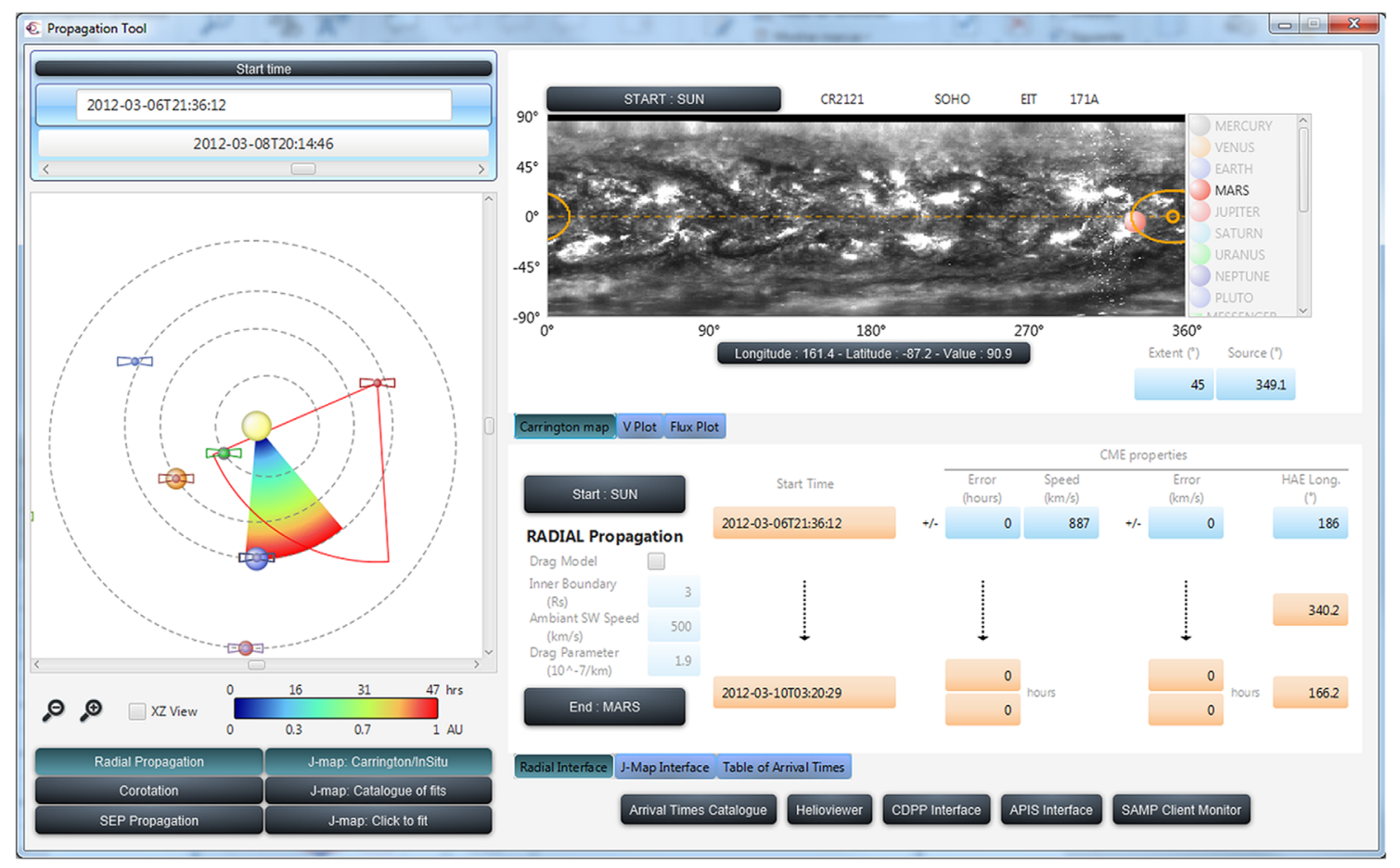

Fig. 3. Screenshot of PROPTOOL. The figure shows the predicted arrival times at Mars of a CME detected on the 6 March 2012. Symbols at different distances from the Sun represent Mercury and Messenger (green); Venus and Venus Express (orange); the Earth and SOHO (Blue); STEREO A (red); STEREO B (blue) and Mars and Mars Express (Red). The field of view of SECCHI in STEREO A is shown in red.

\section{2-03-08T12:00}

(a) Ecliptic plane

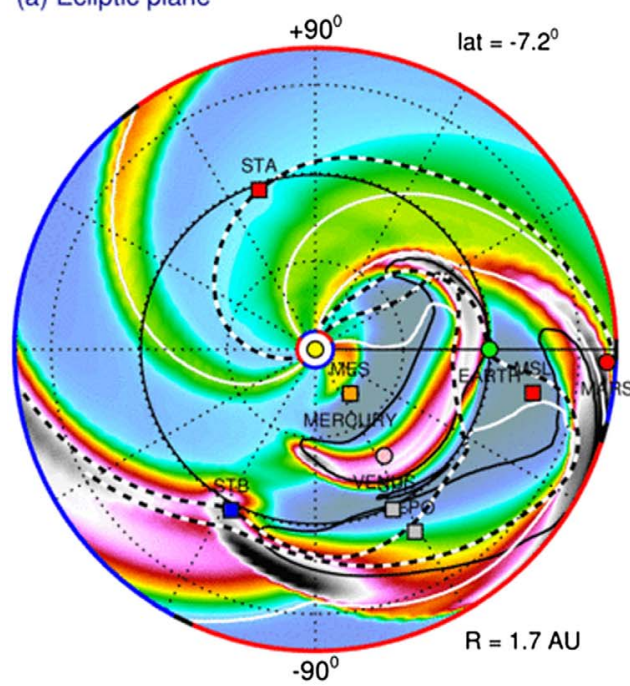

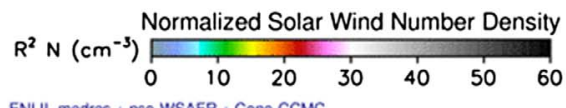

(b) Meridional plane

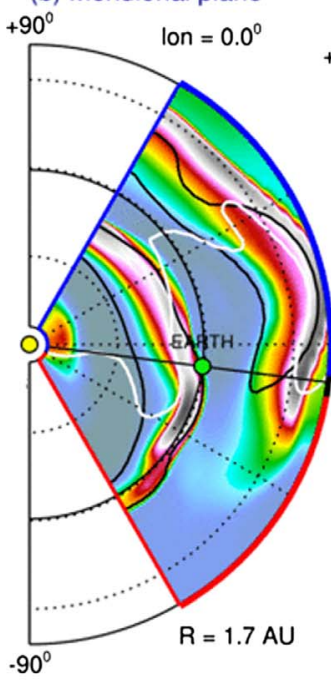

IMF line IMF polarity HCS

2012-03-04T00 + 4.50 days

(c) Radial plane
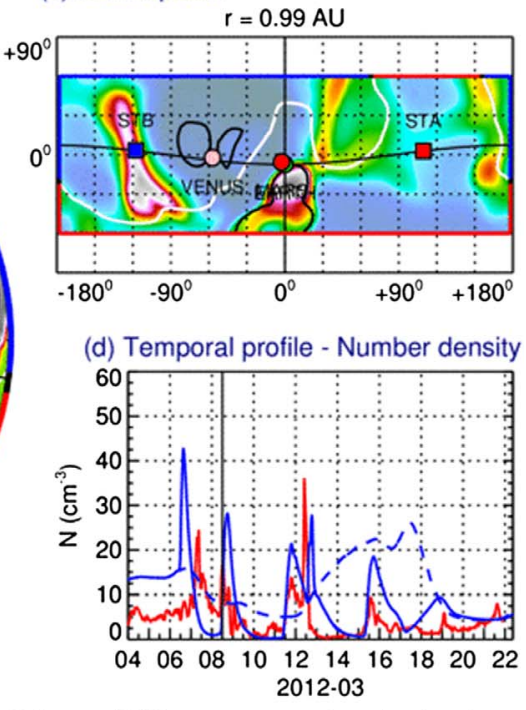

Fig. 4. Example of a run of WSA-Enlil + Cone. Example of a run of WSA-Enlil + Cone showing a simulation of five consecutive CMEs on March 2012. Panel (a) View of the Ecliptic plane; Panel (b) North-South plane including the Earth; Panel (c) Planispheric projection at 1 AU; Panel (d) Comparison of temporal evolution of measured and predicted solar wind particles number density at Earth. 
Table 1. List of online services and propagation tools to study the Sun-Mars connection.

\begin{tabular}{ll}
\hline $\begin{array}{l}\text { Service } \\
\text { acronym }\end{array}$ & Webpage \\
\hline ROB/SIDC & http://sidc.oma.be/ \\
HELSWE & $\begin{array}{l}\text { http://swe.ssa.esa.int/web/guest/metoffice-helswe- } \\
\text { federated }\end{array}$ \\
PROPTOOL & http://propagationtool.cdpp.eu \\
SPWC & $\mathrm{https://www.swpc.noaa.gov/products-and-data}$ \\
iSWA & $\mathrm{https://iswa.gsfc.nasa.gov/IswaSystemWebApp/}$ \\
CCMC & https://ccmc.gsfc.nasa.gov/ \\
\hline
\end{tabular}

large-scale impact in Jupiter in July 2009 (Sánchez-Lavega et al., 2010) produced immediate excitement. Follow-up observations were quickly obtained with telescopes such as the Hubble Space Telescope (HST), the Very Large Telescope (VLT), Gemini, NASA's InfraRed Telescope Facility (IRTF) and Keck. Reports of these observations focused on the dispersal of the debris left by the impact in Jupiter's upper atmosphere (Hammel et al., 2010; Sánchez-Lavega et al., 2010), the nature of the impact body (Orton et al., 2011) and its effects on the local chemistry and temperatures (Fletcher et al., 2010, 2011; Fast et al., 2011). The long-term visibility of the debris was studied with observations from several professional telescopes observing in the near-infrared in strong methane absorption bands (Orton et al., 2011) and with small telescopes and amateur observers observing in the visible and in the methane absorption band at $890 \mathrm{~nm}$ (Sánchez-Lavega et al., 2011).

Figure 5 shows images of the impact material in Jupiter from the original images acquired by A. Wesley to the quick follow-up by HST and a comparison with the continuous study from small size telescopes. The impact cloud material was observable on amateur images until the end of September 2009. These images were acquired in the visible using broadband filters, where the impact features appear as clouds of unusually dark material, but also in the $890 \mathrm{~nm}$ methane absorption band, where impact debris features appear bright due to the altitude of the debris material (about 1-10 mbar). Using large telescopes such as the IRTF in Hawaii, the impact cloud material continued to be observable in the strong hydrogen $\mathrm{H}_{2}-\mathrm{H}_{2}$ collision absorption band at $2.1 \mu \mathrm{m}$ and the strong methane absorption band in $2.3 \mu \mathrm{m}$ images at least until December 2009.

If such an event were observed again in favorable conditions close to Jupiter's opposition, the better sensitivity of

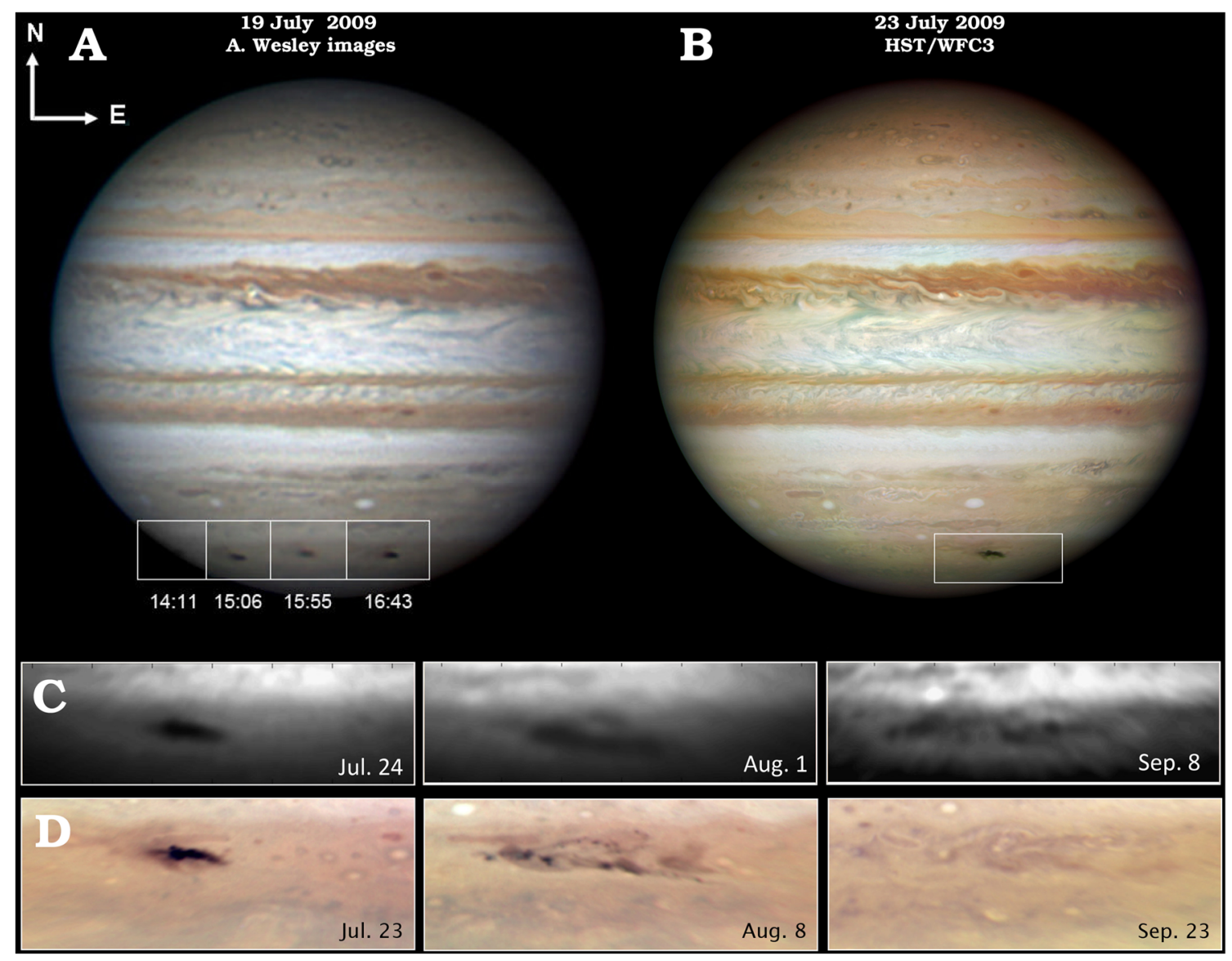

Fig. 5. Debris field on Jupiter from the 2009 impact. (a) Discovery images obtained by Anthony Wesley. (b) HST/WFC3 image of Jupiter showing the impact debris about seven Jupiter rotations later. (c) Series of observations with small-size telescopes of the expansion of the dark debris. (d) Images obtained with HST over similar dates. Data from panel (a) from Sánchez-Lavega et al. (2010). Data from panels (b) and (d) from Hammel et al. (2010). Data from panel (c) from Sánchez-Lavega et al. (2011). 

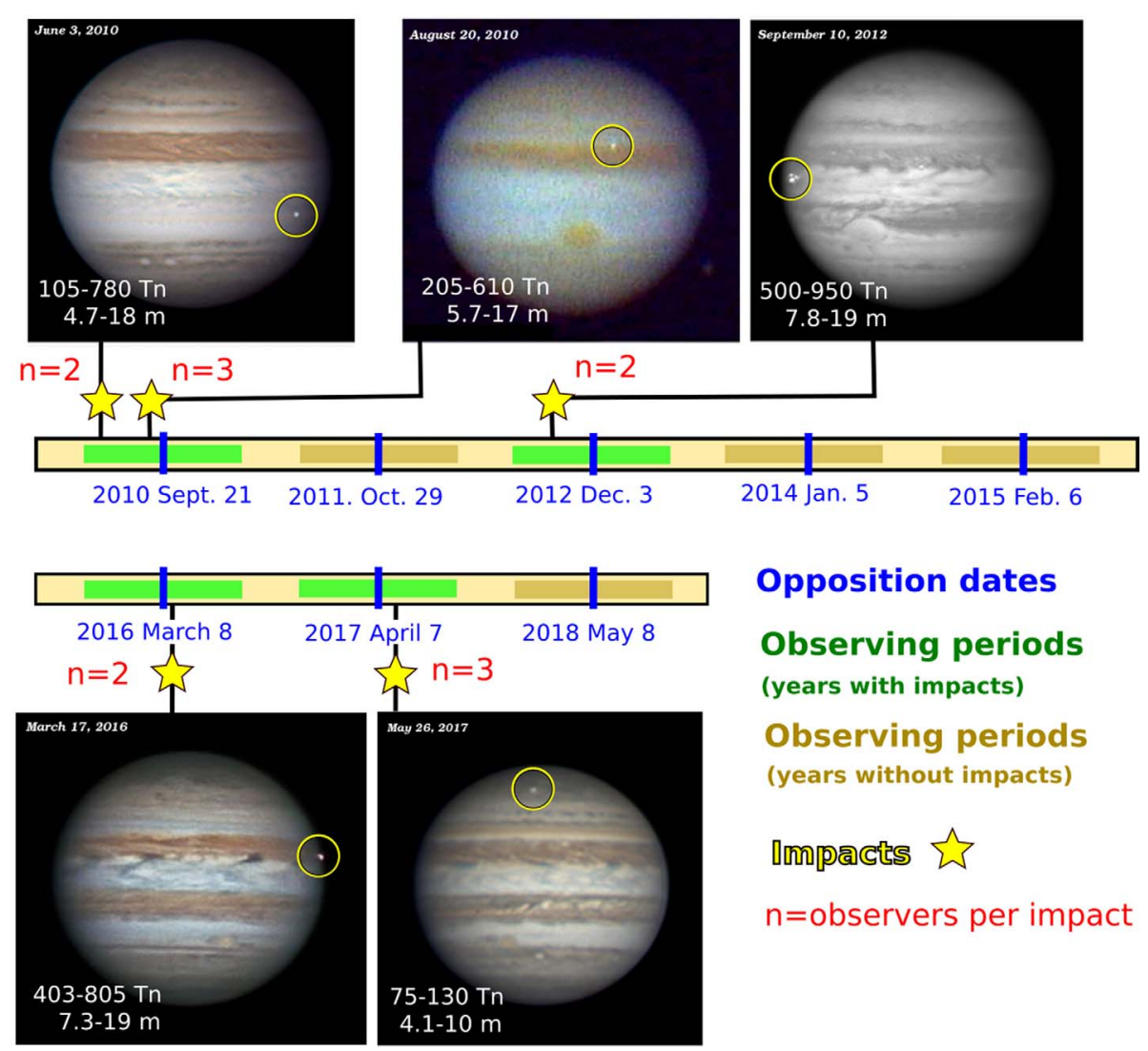

\section{Opposition dates}

Observing periods

(years with impacts)

Observing periods

(years without impacts)

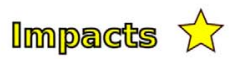

$\mathrm{n}=$ observers per impact

Fig. 6. Timeline of impact flashes on Jupiter. Summary of observations of impact flashes on Jupiter according to data published in Hueso et al. (2018b). Observing periods are centered in each Jupiter opposition and extend about 9 months. The lack of impacts detected in 2014 and 2015 is accompanied by Jupiter oppositions in winter in the Northern hemisphere.

cameras currently used by amateur observers in the near infrared would allow a longer time-line of observations of the debris dispersal using filters centered in the strong methane absorption band at $890 \mathrm{~nm}$ (cameras used by amateur observers since 2016 have increased by a factor of 4 their quantum efficiency in the near infrared when compared with cameras used in 2009). Smaller debris fields produced by smaller and more frequent impacts can also be observable with the current technology and the larger number of amateur observers performing high-quality observations or even using 1-m size telescopes.

An overall comparison of amateur observations of Jupiter from 2009 to 2018 from images archived in the Planetary Virtual Observatory and Laboratory (PVOL) database (Hueso et al., 2010a, 2018a) shows excellent perspectives in this regard. This database contains almost 40000 observations of Solar System planets since the year 2000 and can be consulted online on its own website (http://pvol2.ehu.eus) or through the Virtual European Space Planetary Access (VESPA) query interface (http://vespa.obspm.fr/) (Erard et al., 2018). While PVOL has been designed for scientific use of the data, we acknowledge the existence of similar databases like that of the Association of Lunar and Planetary Observers (ALPO) (http://alpo-j.asahikawa-med.ac.jp/), and many national astronomical databases like that of the Societé Astronomique de France (SAF) (http:// www.astrosurf.com/planetessaf/). An inspection of images in these databases and image repositories shows that, instead of a large increase in quality in the images acquired by the best observers, the main advantage of modern amateur data compared with amateur data obtained 10 years ago are: (i) the better overall quality and the larger number of excellent observers able to attain diffraction-limited images and, (ii) observations acquired by a few excellent observers with access to telescopes with apertures of $50 \mathrm{~cm}$ or larger. Additionally, statistical analysis of data stored in the PVOL database shows that modern amateur observations of Jupiter in 2016-2017 are 2.5 times more numerous in visible wavelengths and 15 times more numerous in the methane absorption filter than in 2009. These methane band images are particularly useful for the discovery and long-term track of impact debris. Considerable uncertainty exists in the current impact rate on Jupiter. Hueso et al. (2018b) present arguments suggesting that dark debris fields on Jupiter's atmosphere from intermediate size objects could be found once every 4-12 years.

\subsection{Jupiter flashes}

Since June 2010 five impact flashes have been found on Jupiter with the last one detected in May 2017 (Hueso et al., 2010b, 2013, 2018b). Each of these impacts has been observed by at least two observers using telescopes with apertures from 12.5 to $37 \mathrm{~cm}$ and generally close to Jupiter's opposition. Figure 6 shows a summary of these impact flashes and Table 2 
Table 2. Summary of flashing impacts in Jupiter (adapted from Hueso et al., 2018b).

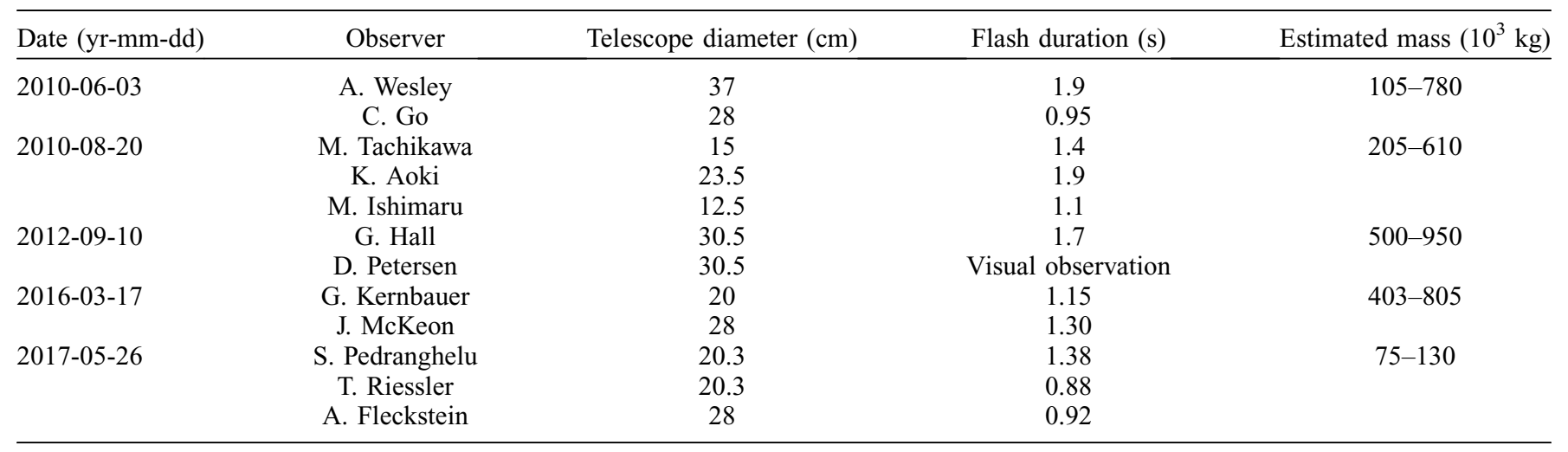

summarizes the masses attributed to these objects and the telescopes used for their discovery. Figure 7 shows the different detections as a function of telescope diameter and as a function of time. Bigger telescopes should be able to detect fainter impacts, but the current statistics is so small that a general trend in this relation cannot be observed. However, a trend towards the increasing capability of detecting smaller flashes on recent years seems apparent from this figure.

Hueso et al. $(2013,2018 b)$ present a comparative analysis of the properties of these impacts and calculate the statistics of these events predicting impact rates for similar objects (diameters from 5-20 m depending on the density and masses from Table 2) on Jupiter on the range of 10-65 impacts per year. However, even if we could observe the planet almost continuously from the Earth, only 4-25 of these impacts would be potentially observable as flashing impacts. This is so because of the observational cycle of Jupiter that extends about 9 months per year at best, and because impacts on the occulted side of the planet cannot be observed. Therefore, further discoveries of flashing impacts in Jupiter will require a considerable amount of observational time and serendipitous discoveries by the global community of amateur astronomers is the best method to perform new impact detections.

There is an additional difficulty in detecting Jovian flashing impacts. Even if Jupiter is observed while an impact happens, and a video of the event is recorded with a reasonable signal to noise ratio, it may still skip detection. The reason is that most amateur observers obtain their video observations over long observing sessions in order to build "stacked" images of the planet that can be processed to build high-resolution images (Mousis et al., 2014; Hueso et al., 2018a). The task of transforming a video into a final images is generally performed by automatic software tools that stack minutes of data (Kraaikamp, 2016) erasing the potential signal of a short-time visible impact in the final image. Additionally, only a small proportion of amateurs carefully check their video observations while they are running a long observation run. Experience from the previous five flashes shows that, in each case, only a single observer detected each impact, either while performing his own observation, or during a later analysis of their video. Confirming videos for each impact only came out when other observers heard about the impact and found the impact on their own video data of the same observing instant knowing when the impact happened. Thus, the "success rate" of these positive detections is five impacts over 12 observations or $42 \%$ but could be lower if more impacts have been recorded and passed unnoticed. Noticeably, the brightest flash recorded (the Sept. 2012 impact recorded by George Hall, an observer from USA) was not initially detected in the video observation where it was later found, but it was first announced by Dan Petersen, a visual observer who was looking at the planet through its eyepiece that night (see Hueso et al., 2013 for details). This means that adequate software tools released to the amateur community may improve the detectability of these events.

\section{Detection software}

Impacts can be automatically detected using differential photometry algorithms in which each video frame is compared with a reference image showing the impact location. Software tools aimed to detect impacts exist indeed for the analysis of videos of the non-illuminated observable part of the Moon like the MIDAS software (Madiedo et al., 2015). However, Jupiter observations have different requirements (large magnification and strong influence of the atmospheric seeing, bright background in the pixels where the impact happen, large variety of acquisition cameras and video formats). While it would be almost impossible to set-up a server where individual observers could upload their raw observations for analysis (that would require a large infrastructure capable of analyzing hundreds of Terabytes of data), we have developed an open source software tool called DeTeCt that performs this task and that can be distributed to individual observers willing to perform the search of impacts. Because of historical reasons there are two versions of the software being used by the amateur community DeTeCt 2 (a Windows/Linux application running from the command line) and DeTeCt3.1 (a Windows application with a Graphical User Interface that has been developed to make the software easier to use for a wider audience). The development of DeTeCt3.1 has been one of the virtual activities of the Planetary Space Weather Services (PSWS) offered by the Europlanet H2020 Research Infrastructure (André et al., 2018). Both software tools can analyze hundreds of video files recursively from a specified folder and both run essentially the same detection algorithm. In both cases, they produce "detection images" and log files that 

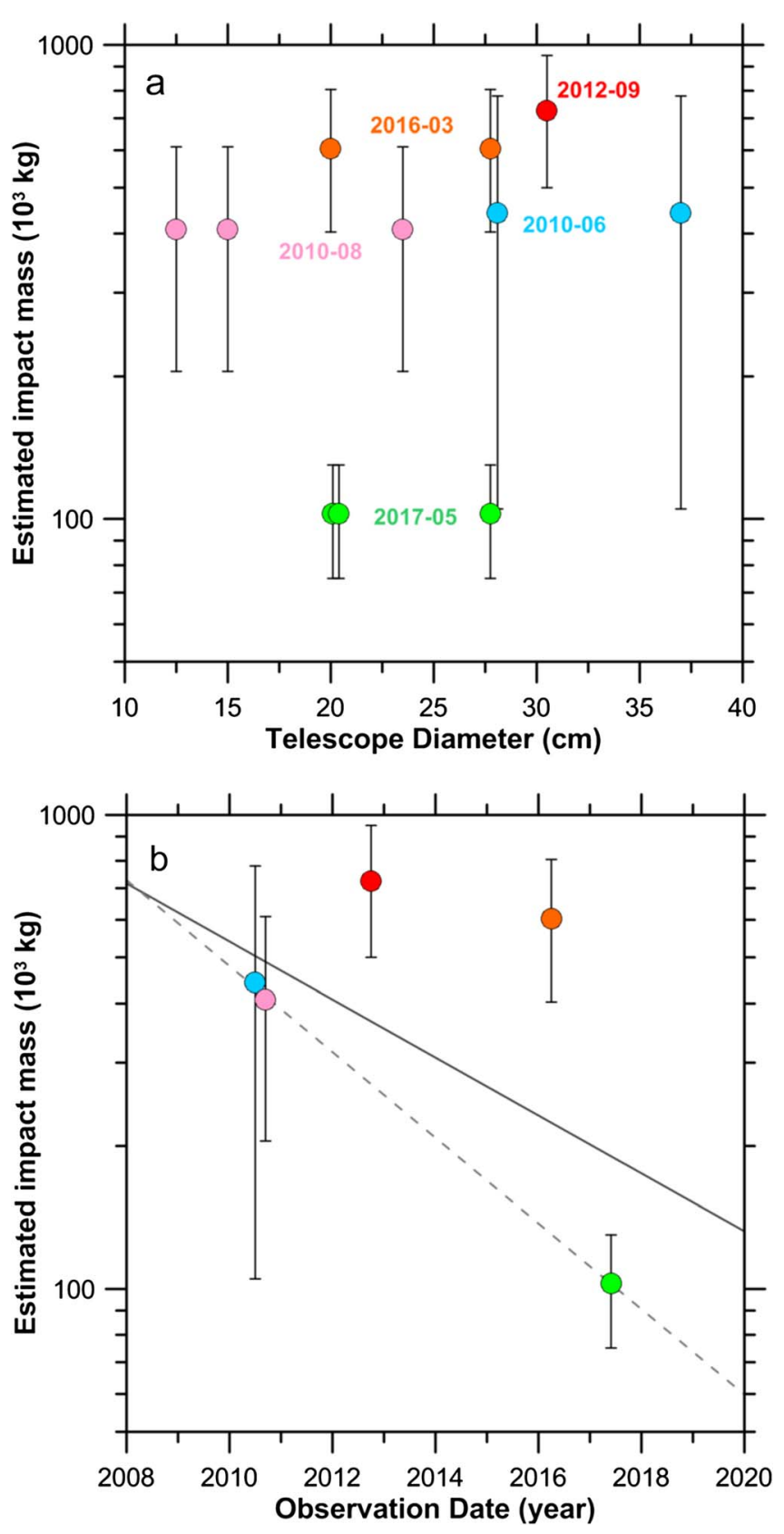

Fig. 7. Estimated masses of impact objects in Jupiter. (a) As a function of the telescope diameter used in the detection, (b) As a function of the time of the discovery. Solid and dashed lines represent possible fits to the ensemble of the data and the minimum detected masses respectively.

are sent to one of us (M.D.) for statistical analysis of the nondetections. Updated results are published online on: http:// www.astrosurf.com/planetessaf/doc/project_detect.shtml

Considerable efforts were put in the software to analyze the large range of video formats produced by cameras used by amateur astronomers. The software and its source code are available at the PVOL database (http://pvol2.ehu.es/psws/ jovian_impacts) together with background scientific information and a software guide with detailed instructions. The source code is also available in the github platform with the project name: "DeTeCt-PSWS" under license GNU AGPLv3 (see https://github.com/DeTeCt-PSWS/DeTeCt-MFC). The software is open source, documented, and can be modified accordingly.

We now give a summarized description of the algorithms and steps followed by DeTeCt to analyze video observations and find short flashes of light. Because video observations of Jupiter are affected by the quality of the equipment used and the quality of the atmospheric seeing, designing a software tool that accommodates to a vast variety of video properties is difficult and the software produces some false positives. For this reason, the software accommodates two strategies that run in parallel: a detection algorithm, and the built of a detection image for each video that can be quickly inspected by a human user. Users of the software can find practical information in a user guide describing the interface and the configurable parameters in the software. Figure 8 summarizes the steps followed by the software in a graphical way.

1. Video coregistration: Each video is analyzed first finding the planet's location on the first frame of the video. This frame is used to define a ROI containing the planet through the calculation of the center of brightness (CB) of the image. This planet location works better after subtracting an average background calculated from the borders of the image. The definition of this ROI is shown in Figure 9. The CB location is used to obtain vertical and horizontal photometric cuts that define the size of the planet through the use of a convenient brightness threshold (red lines on panels b and c in Fig. 9) and a security margin. The $\mathrm{CB}$ is identified in subsequent frames shifting each frame to coregister them with the initial frame. This procedure can produce small errors in the coregistration of different frames. In blurred frames, the errors can as high as 10 pixels. For this reason, a second step is to compute the final shifts for the best coregistration between the analyzed frame and the first one using a two-dimensional correlation algorithm.

2. Differential photometry: A reference "stacked" image is built as the video file is read and from the coregistered frames. The last $N$ frames are used for this purpose where $N$ can be defined by the user and is generally 50 frames. If the software is analyzing the first frames of the video the reference is built with a lower number of the frames preceding the one being analyzed. Each frame is normalized in brightness with respect to the first one to avoid brightness variations produced by passing high cirrus. For each frame, a differential photometry image is built by subtracting the last reference. Differential images are noisy and have their highest values generally concentrated on the limb of the planet where the planet shape continuously distorts by varying seeing. An impact would appear as a region of closely packed bright pixels. To avoid spurious detections produced by hot pixels or pixel noise we apply a median filter to the differential photometry image and a small smoothing factor. The radius of these filters has been optimized after extensive testing from the 11 video files where impacts have been detected in the past. After these smoothing steps the impact region generally contains the brightest pixels in the differential images. 


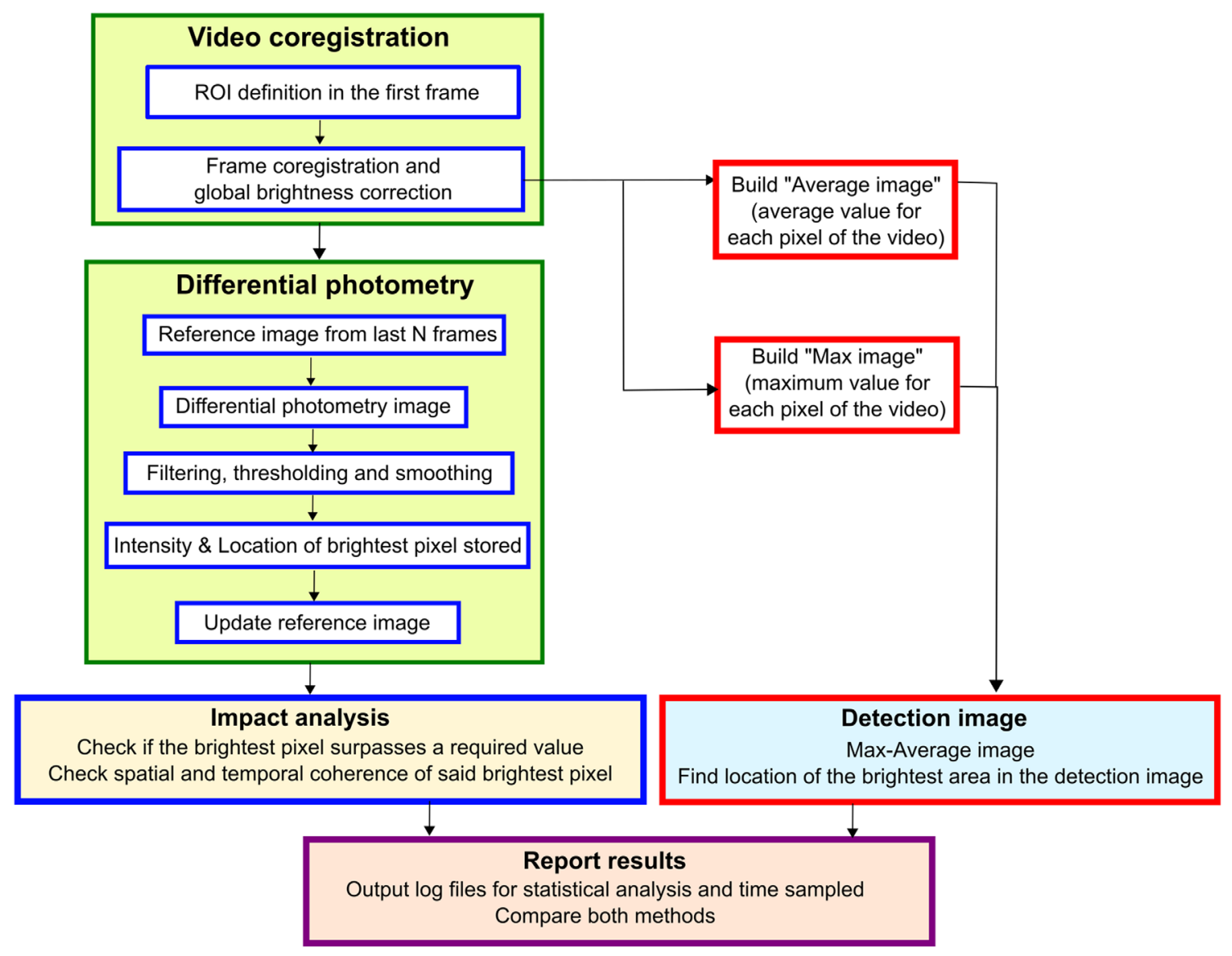

Fig. 8. DeTeCt algorithm. Main steps involved in video processing and flash detection.

3. Analysis: For each differential photometry frame the value of the brightest pixel and its location are stored in a list. After the video has been fully analyzed the software examines this list looking for differential values higher than a minimum threshold and with $x-y$ coordinates closer than a 10 pixel difference. These data are analyzed by the software to produce a detection message. Figure 10 shows an example of these data for the Sept. 2012 impact.

4. Detection image: In parallel to these steps DeTeCt builds a "detection image" that should be checked visually by the software user. This image is built from coregistered frames. For each pixel, the difference between the brightest value at that location and its average is produced. The detection image is analyzed by the software looking for its brightest location. Figure 8 shows outputs from the software in the form of detection images over a variety of video observations. False negative detections are not covered in this figure but generally concentrate in the limb of the planet (like in panel $\mathrm{e}$ in Fig. 11) and in satellites or satellite shadows over the planet.

In bright impacts both detection methods find the impact at the same location but in weak impacts, or in videos obtained under a bad atmospheric seeing, they may produce different results. Detection images can be examined by the user to identify false positive detections and the software user guide contains several examples of detection images. Figure 11 shows some of these examples for detections of true impacts in the planet summarized in Table 2. Figures $11 \mathrm{a}-11 \mathrm{c}, 11 \mathrm{f}$ and $11 \mathrm{~g}$ show clear detections of impacts considered bright from their derived properties (see Table 2). Figures 11d and $11 \mathrm{e}$ shows examples of analyses where the detection image shows the presence of an impact unambiguously but the temporal analysis of the photometry does not. Furthermore, some video files have low quality and the software is not able to find an impact even if a true impact can be observed in the video file (Fig. 11e). However, detection images are always a quick way to determine if a possible event is present in the video file or not. Success rates for the 11 video recording are: $55 \%$ for the detection algorithm and $90 \%$ for the automatic analysis of detection images. Direct observation of the videos is only accurate if the observer is willing to spend his time reviewing observations. However, the main advantage of the DeTeCt software is that it can be used without any interaction from the user to analyze large collections of video files. Thus, the user should only spend his time reviewing the detection images and $\log$ files. The use of the software by a large number of amateurs may result in detections of new impacts but also of 

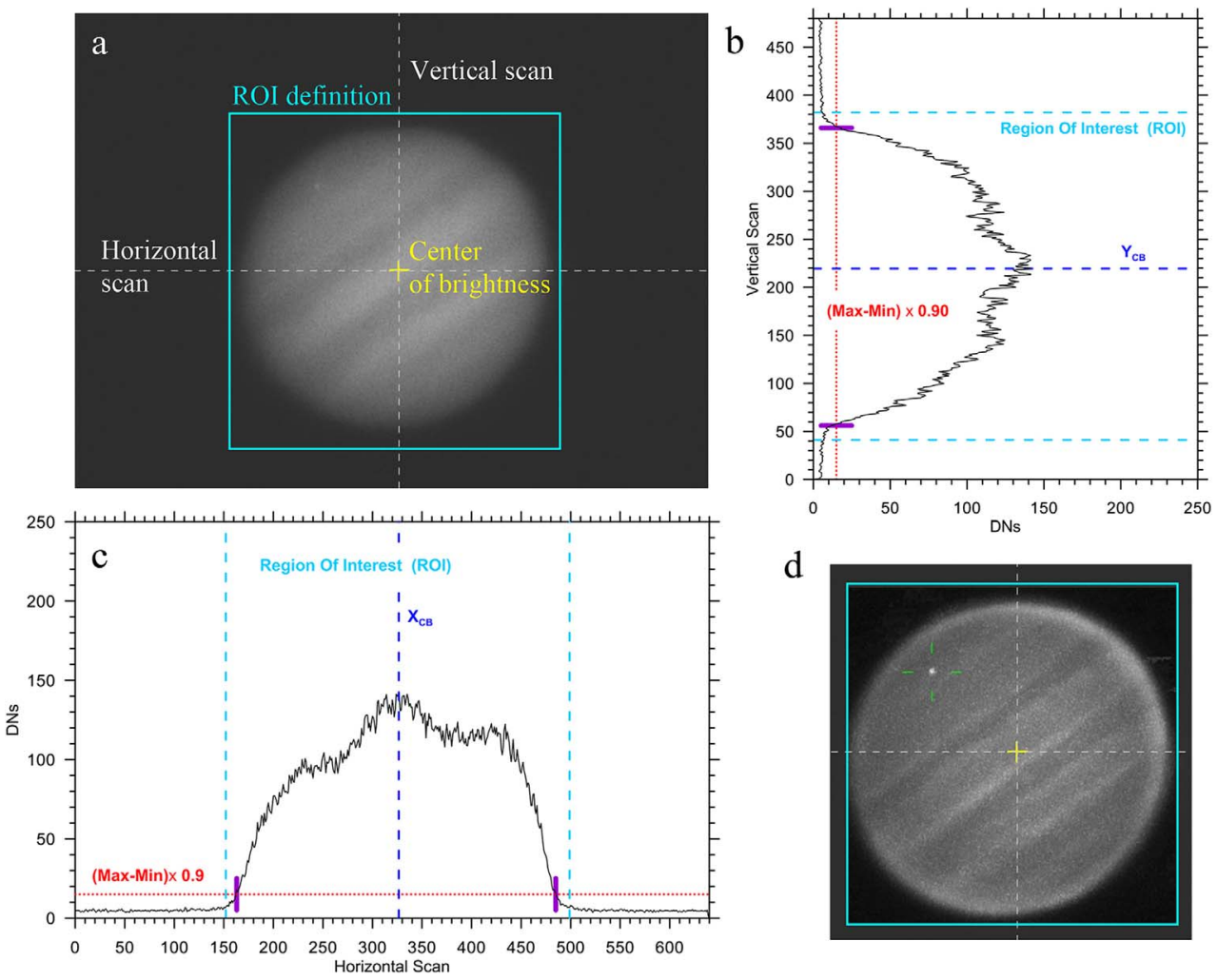

Fig. 9. Detection algorithm. (a) The first frame of the video is used to define a ROI using the CB of the image and vertical and horizontal scans displayed on panels (b) and (c). A given threshold with a margin allows to compute a box surrounding the planet. Panel (d) shows a differential photometry image produced by the software where the impact location is highlighted with a green cross.

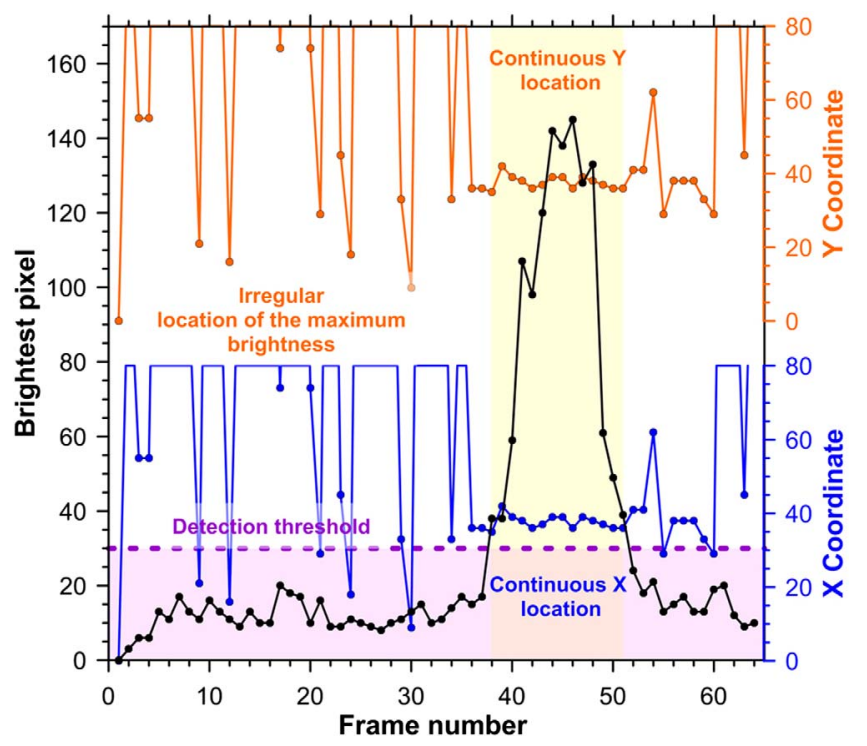

Fig. 10. Analysis of the photometry data. The brightest pixel on the differential image (black line and dots, left axis) is compared with respect to a detection threshold (dashed magenta line). For an impact to be detected the brightest region of this curve must also verify that the location of the brightest pixel is nearly continuous (blue and orange line on the right axis). The impact is detected by the software in the region highlighted in yellow. Example is for the September 2012 impact. old impacts that were not discovered at the time of the observation.

\section{Impacts detection campaigns and global analysis}

Current observations are carried out by several collaborators. Although up to now none of the discovered impact flashes has been detected by running this software we recall that the software is only being used by a small percent of the amateur community and we are working towards a wider use of DeTeCt. Both versions of the DeTeCt software produce short text $\log$ files with timing of the videos that are sent to one of us (M.D.) for statistical analysis. This analysis is done by a set of software scripts that we use to know how much time the network of collaborators are observing Jupiter in search for impacts. Currently, the software has been used by 78 observers examining more than 76800 video files equivalent to about 87 days of observations distributed unevenly over the last few years. A similar survey of Saturn videos with 17 observers has accumulated a total observation time equivalent to 11.4 days with negative results in the search of impacts on Saturn so far. Updates of these statistics are reported on: http://www.astrosurf.com/planetessaf/doc/project_ detect.shtml. We expect that users of the software will be far more numerous in the near future since DeTeCt3.1 (which is 

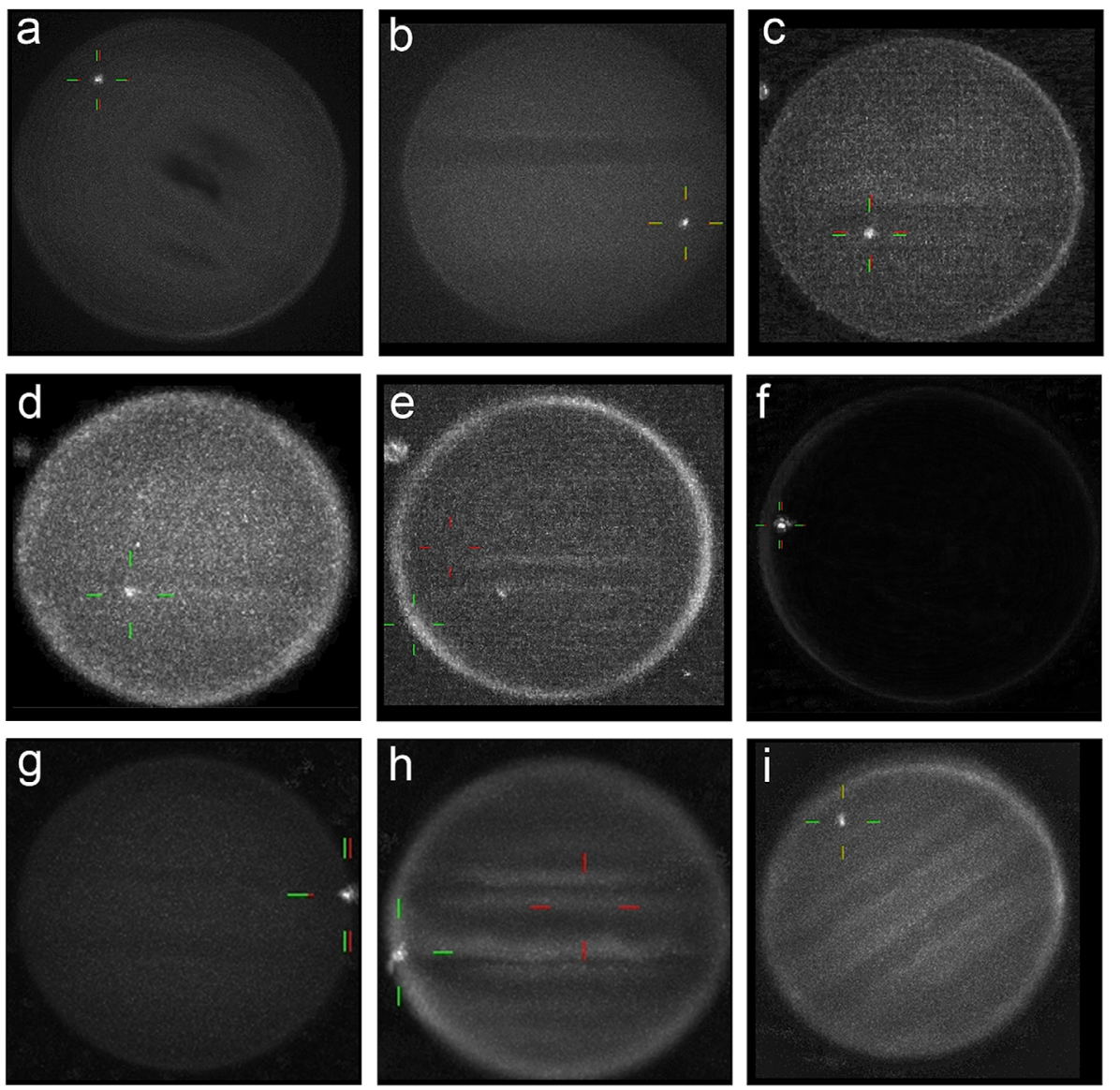

Fig. 11. Detection images produced by the software. Detection images from the analysis with DeTeCt3.1 of video observation of true impacts by (a) A.W., (b) C.G., (c) M.T., (d) M.I., (e) K.A., (f) G.H., (g) J.McK., (h) G.K., (i) T.R. Red-crosses are automatically generated by the software highlighting the impact detected in the photometry analysis. Green-crosses are automatically generated by the software analyzing the detection images. In robust detections, they point to the same location but sometimes only, the detection image finds the impact (h). In some cases of weak impacts or lower quality videos, none of the two methods find the impact (e) but a trained eye can distinguish a true impact in the detection image.

more user friendly than the previous version) was only released on March 2018.

\section{Perspectives and conclusions}

The last Mars opposition in July 2018 attracted a high interest of the amateur observers due to the favorable conditions of the opposition with Mars reaching 24.3 arcsec in diameter. This opposition occurred at solar minimum, but the next two Mars oppositions in October 2020 and December 2022 will also result in high-quality observations of the planet due to its large apparent size (Fig. 2). The solar activity will be close to maximum in December 2022 with a declination of $+25^{\circ}$ favoring observers from North latitudes where a higher number of amateur observers reside. In the case of a CME with a high probability to arrive to Mars, a quick alert can be issued to amateurs through popular sites such as the PVOL database where regular alerts on Jupiter, Saturn and Neptune meteorological features are issued.

Amateur astronomers are actively searching impacts in Jupiter (Hueso et al., 2018a) and Saturn using DeTeCt.
A related field is the search of smaller-size flash impacts in the Moon (Madiedo et al., 2014; Ortiz et al., 2015) where amateurs can largely contribute. Impacts on Mars seem not possible to detect from ground-based observations. Fresh craters on Mars discovered by different Mars orbiters (Daubar et al., 2013, 2014) could be produced by objects hitting the planet with about 200 times less energy than Jovian impacts detected by amateur observers. The impact rate on Mars (Stevanocić et al., 2017) suggests that flashes impact on Mars with energies releases 10 times lower than those observed on Jupiter (and hence, still potentially observable) would only appear once every 80 years at most making this possibility an extremely unlikely discovery.

Existing data stored in the archives of the global amateur community may contain other events of high-atmospheric structures on Mars upper atmosphere or non-detected impacts in Jupiter or Saturn. This can be the case especially for marginal detections in the limit of the quality of the original observations. However, as technology improves (low-cost more sensitive faster cameras) and amateurs become more aware of these phenomena the chances of non-detecting future events will diminish. Higher quality images of Mars will ease the 
search of high-atmospheric phenomena, and impacts produced by smaller objects impacting Jupiter more often will be observable with small telescopes. The brightest impact flashes of the next few years will be better characterized from the high cadence of the observations that are possible now (many amateurs currently record videos of Jupiter at 60 frames per second). For Saturn we have never witnessed a direct impact in the planet, but the consequences of small impacts in the rings have been observed with Cassini images (Tiscareno et al., 2013) and the long-term effect of large impacts on Saturn's rings has also been observed (Hedman et al., 2011). Impacts produced by objects roughly five times more massive than those producing flashes on Jupiter could produce flashes on Saturn that would be detectable with telescopes used by amateur astronomers and their range of detectors. Unfortunately, Saturn is a more difficult target, it attracts a smaller number of observers and, generally, only advanced amateurs produce high-quality observations of this planet. Since the impact rate in Saturn is still less constrained than that of Jupiter it is difficult to estimate the chances of finding similar flashes on this planet. However, these chances continue to increase and, eventually, a Saturn flash impact will be discovered.

In both science cases, amateur observers play a pivotal role and will continue to do so in the near future.

Acknowledgements. We thank Dave Andrews and an anonymous referee for their review of this paper. We are grateful to Ishi Tabe in Japan and C. Palotai at Florida Institute of Technology and Kunio Sayanagi at Hampton University for motivating further observations of Jupiter oriented to find new impacts. We are also specially grateful to the planetary astronomy amateur community for their continuous observations of Solar System planets and in particular to users of DeTeCt collaborating with this project. Part of this work has been developed in the framework of the Europlanet 2020 RI, which has received funding from the European Union's Horizon 2020 research and innovation programme under grant agreement No 654208. Further support was given by the Spanish projects AYA2015-65041-P (MINECO/FEDER, UE) and Grupos Gobierno Vasco IT- 765-13. The editor thanks Dave Andrews and an anonymous referee for their assistance in evaluating this paper.

\section{References}

André N, Grande M, Achilleos N, Barthélémy M, Bouchemit M, et al. 2018. Virtual planetary space weather services offered by the Europlanet H2020 research infrastructure. Planet Space Sci 150: 50-59.

Andrews DJ, et al. 2016. Plasma observations during the Mars atmospheric "plume" event on March-April 2012. J Geophys Res Space Phys 121: 3139-3154.

Andersson L, Weber TD, Malaspina D, Crary F, Ergun RE, et al. 2015. Dust observations at orbital altitudes surrounding Mars. Science 350: aad0398.

Barabash S, Lundin R, Andersson H, Brinkfeldt K, Grigoriev A, et al. 2006. The Analyzer of Space Plasmas and Energetic Atoms (ASPERA-3) for the Mars express mission. Space Sci Rev 126: 113-164.
Clancy RT, Wolff MJ, Whitney BA, Cantor BA, Smith MD. 2007. Mars equatorial mesospheric clouds: Global occurrence and physical properties from Mars Global Surveyor Thermal Emission Spectrometer and Mars Orbiter Camera limb observations. $J$ Geophys Res 112(E4): E04004.

Clancy RT, Montmessin FB, Benson J, Daerden F, Colaprete A, Wolff MJ. 2017. Mars clouds. In: The atmosphere and climate of Mars, Haberle RM, (Ed.), Cambridge University Press.

Daubar IJ, McEwen AS, Byrne S, Kennedy MR, Ivanov B. 2013. The current Martian cratering rate. Icarus 225: 506-516.

Daubar IJ, Atwood-Stone C, Byrne S, McEwen AS, Russell PS, et al. 2014. The morphology of fresh craters on Mars and the Moon. J Geophys Res Planet 119: 2260-2639.

de Pater I, Fletcher LN, Pérez-Hoyos S, Hammel HB, Orton GS, Wong MH, Luszcz-Cook S, Sánchez-Lavega A, Boslough M. 2010. A multi-wavelength study of the 2009 impact on Jupiter: Comparison of high resolution images from Gemini, Keck and HST. Icarus 210: 722-741.

Duru F, et al. 2017. Response of the Martian ionosphere to solar activity including SEPs and ICMEs in a two-week period starting on 25 February 2015. Planet Space Sci 145: 28-37.

Erard S, Cecconi B, Le Sidaner P, Rossi AP, et al. 2018. VESPA: A community-driven virtual observatory in Planetary Science. Planet Space Sci 150: 65-85.

Fast KE, Kostiuk T, Livengood TA, Hewagama T, Annen J. 2011. Modification of Jupiter's stratosphere three weeks after the 2009 impact. Icarus 213: 195-200.

Fletcher LN, Orton GS, de Pater I, Mousis O. 2010. Jupiter's stratospheric hydrocarbons and temperatures after the July 2009 impact from VLT infrared spectroscopy. $A \& A$ 524(A46): 14.

Fletcher LN, Orton GS, de Pater I, Edwards ML, Yanamandra-Fisher PA, Hammel HB, Lisse C, Fisher BM. 2011. The aftermath of the July 2009 impact on Jupiter: Ammonia, temperatures and particulates from Gemini thermal infrared spectroscopy. Icarus 211: 568-586.

Gurnett DA, Kirchner DL, Huff RL, Morgan DD, Persoon AM, et al. 2005. Radar soundings of the ionosphere of Mars. Science 310: 1929-1933.

Hammel HB, Beebe RF, Ingersoll AP, Orton GS, Mills JR, et al. 1995. HST imaging of atmospheric phenomena created by the impact of Comet Shoemaker-Levy 9. Science 267: 1288-1296.

Hammel HB, Wong MH, Clarke JT, de Pater I, Fletcher LN, et al. 2010. Jupiter after the 2009 impact: Hubble space telescope imaging of the impact generated debris and its temporal evolution. Astrophys J Lett 715: L150-L154.

Harrington J, de Pater I, Brecht SH, Drake D, Meadows V, Zahnle K, Nicholson PD. 2004. Lessons from Shoemaker-Levy 9 about Jupiter and planetary impacts. In: Jupiter: The planet, satellites and magnetosphere, Bagenal F, Downling TE, McKinnon WB (Eds.), Cambridge University Press, Cambridge, United Kingdom, pp. $159-1184$.

Hedman MM, Burns JA, Evans MW, Tiscareno MS, Porco CC. 2011. Saturn's curiously corrugated C ring. Science 332: 708.

Hueso R, Legarreta J, Pérez-Hoyos S, Rojas JF, Sánchez-Lavega A, Morgado A. 2010a. The International Outer Planet Watch atmospheres node database of giant-planet images. Planet Space Sci 58: 1152-1159.

Hueso R, Wesley A, Go C, Pérez-Hoyos S, Wong MH, Fletcher LN, Sánchez-Lavega A, et al. 2010b. First Earth-based detection of a Superbolide on Jupiter. Astrophys J Lett 721(2): L129.

Hueso R, Pérez-Hoyos S, Sánchez-Lavega A, Wesley A, Hall G, Go C, et al. 2013. Impact flux on Jupiter: From superbolides to large-scale collisions. $A \& A$ 560: A55. 
Hueso R, Juaristi J, Legarreta J, Sánchez-Lavega A, Rojas JF, Erard S, Cecconi B, Le Sidaner P. 2018a. The planetary virtual observatory and laboratory (PVOL) and its integration into the virtual European solar and planetary access (VESPA). Planet Space Sci 150: 22-35.

Hueso R, Delcroix M, Sánchez-Lavega A, et al. 2018b. Small impacts on the Giant Planet Jupiter. $A \& A$ 617: A68.

Jaquin F, Gierasch P, Kahn R. 1986. The vertical structure of limb hazes in the Martian atmosphere. Icarus 68: 442-461.

Kraaikamp E. 2016. Sky and telescope, vol. 132, Sky publishing Corporation, Cambridge, USA, p. 68.

Law NM, Mackay CD, Baldwin JE. 2006. Lucky imaging: high angular resolution imaging in the visible from the ground. $A \& A$ 446: 739-745.

Madiedo JM, Ortiz JL, Morales N, Cabrera-Caño J. 2014. A large lunar impact blast on 2013 September 11. MNRAS 439: 2364-2369.

Madiedo JM, Ortiz JL, Morales N, Cabrera-Caño J. 2015. MIDAS: Software for the detection and analysis of lunar impact flashes. Planet Space Sci 111: 105-115.

Mendikoa I, Sánchez-Lavega A, Pérez-Hoyos S, Hueso R, Rojas JF, Aceituno J, Aceituno F, Murga G, de Bilbao L, García-Melendo E. 2014. PlanetCam UPV/EHU: A two channel lucky imaging camera for Solar System studies in the spectral range 0.38-1.7 microns. Publ Astron Soc Pac 128: 035002 (22 p.).

Minami M. 2003. Communication in Mars observations, vol. 283. http://www.kwasan.kyoto-u.ac.jp/ cmo/cmomn2/283OAA/ index.htm.

Mitchell DL, Lillis RJ, Lin RP, Connerney JEP, Acuña MH. 2007. A global map of Mars' crustal magnetic field based on electron reflectometry. J Geophys Res 112: E01002.

Mousis O, Hueso R, Bouley S, Colas F, et al. 2014. Instrumental methods for professional and amateur collaborations in planetary astronomy. Exp Astron 38: 91-191.

Odstrcil D. 2003. Modeling 3-D solar wind structure. Adv Space Res 32: 497-506.

Ortiz JL, Madiedo JM, Morales N, Santos-Sanz P, Aceituno FJ. 2015. Lunar impact flashes from Geminids: Analysis of luminous efficiencies and flux of large meteoroids on Earth. MNRAS 454: 344-352.

Orton GS, Fletcher LN, Lisse CM, Chodas PW, Cheng A. 2011. The atmospheric influence, size and possible asteroidal nature of the July 2009 Jupiter impactor. Icarus 211: 587-602.

Pellier C. 2012. Martian terminator projections observed by the HST. Commun Mars Observ 400: http://www.kwasan.kyoto-u.ac. jp/ cmo/cmomn4/CMO400.pdf.
Picardi G, et al. 2004. MARSIS: Mars advanced radar for subsurface and ionosphere sounding. In: Mars Express: The Scientific Payload, ESA Spec. Publ. 1240, Wilson A, Chicarro A (Eds.), ESA, Noordwijk, Netherlands. pp. 51-69.

Pizzo V, Millward G, Parsons A, Biesecker D, Hill S, Odstrcil D. 2011. Wang-Sheeley-Arge-Enlil cone model transitions to operations. Space Weather 9: 03004.

Pujic Z. 1994. Amateurs observe comet impacts, Southern Sky, Sept/ Oct 1994.

Rouillard AP, Lavraud B., Genot V., Bouchemit M., Dufourg N., et al. 2017. A propagation tool to connect remote-sensing observations with in-situ measurements of heliospheric structures. Planet Space Sci 147: 61-77.

Sánchez-Lavega A, Lecacheux J, Colas F, Gómez-Forrellad JM, Laques P, Miyazaki I, Parker D. 1995. Motions of the SL9 impact clouds. Geophys Res Lett 22: 1761-1764.

Sánchez-Lavega A, Wesley A, Orton GS, Hueso R, Pérez-Hoyos S, Fletcher LN, et al. 2010. The impact of a large object on Jupiter in 2009 July. Astrophys J Lett 715: L155-L159.

Sánchez-Lavega A, García-Muñoz A, García-Melendo E, Pérez-Hoyos S, Gómez-Forrellad JM, Pellier JM, et al. 2015. An extremely high altitude plume seen at Mars morning terminator. Nature 518: 525-528.

Sánchez-Lavega A, Chen-Chen H, Ordonez-Etxeberria I, Hueso R, del Rio-Gaztelurrutia T, Garro A, Cardesín-Moinelo A, Titov D, Wood S. 2018. Limb clouds and dust on Mars from images obtained by the Visual Monitoring Cameraa (VMC) onboard Mars express. Icarus 299: 194-205.

Santer R, Deschamps M, Ksanfomaliti LV, Dollfus A. 1986. Photopolarimetry of Martian aerosols. II - Limb and terminator measurements. $A \& A$ 158: 247-258.

Stevanocić J, Teanby NA, Wookey J, Selby N, Daubar IJ, Vaubaillon J, Garcia R. 2017. Bolide Airbursts as a seismic source for the 2018 Mars insight mission. Space Sci Rev 211: 525-545.

Thampi SV, Krishnaprasad C, Bhardwaj A, Lee Y, Choudhary RK, Pant TK. 2018. MAVEN Observations of the response of Martian ionosphere to the interplanetary coronal mass ejections of March 2015. J Geophys Res Space Phys 123: 6917-6923.

Tiscareno M, Mitchell CJ, Murray CD, Di Nino D, Hedman MM, Jürgen S, Burns JA, Cuzzi JN, Porco CC, Beurke K, Evans MW. 2013. Observations of Ejecta clouds produced by impacts on Saturn's rings. Science 340: 460-464.

Wolff MJ, López-Valverde M, Madeleine JB, Wilson RJ, Smith MD, Fouchet T, Delory GT. 2017. Radiative process: Techniques and applications. In: The Atmosphere and Climate of Mars, Haberle RM (Eds.), Cambridge University Press.

Cite this article as: Hueso R, del Río-Gaztelurrutia T, Sánchez-Lavega A, Delcroix M, Juaristi J, et al. 2018. Detectability of possible space weather effects on Mars upper atmosphere and meteor impacts in Jupiter and Saturn with small telescopes. J. Space Weather Space Clim. 8, A57. 